\title{
Building a long-term relationship
}

\author{
We explore some of the reasons why physicists from Asia are under-represented in Nature \\ Reviews Physics as authors and referees and ponder what we can do.
}

In our January editorial, we explored the geographical diversity of our authors and referees and noticed that Asian scientists are insufficiently represented, especially in the peer-review process. To understand why, as editors, we are failing to engage more with physicists from Asia, we asked nine scientists, editors and science communicators their opinion about the visibility challenges faced by Asian scientists. Their answers, published in a Viewpoint in this Issue, highlight a complex interplay of cultural, social and historical factors, and reinforce the need to better understand the context before trying to find solutions. There are no quick fixes but we want to do what we can.

According to the Nature Index, in the physical sciences, China, Japan and South Korea are ranked in the top 10 in terms of research output. Even if restricted to the physics articles, the numbers are impossible to ignore: physicists in these regions, most notably South Korea (with only one peer reviewer and no corresponding authors), are under-represented in our journal.

This trend may be linked to the idiosyncrasies of research in these countries. For example, in some parts of Asia, applied research is a strong area of focus. In particular, as highlighted in the Viewpoint, in South Korea the funding structure is intended to promote short-term projects with applied outputs relevant for industry, work that does not necessarily fall within the scope of Nature Reviews Physics. This approach to funding stems from the close relationship between research and the post-Korean War fast economic development in South Korea. There has been investment in fundamental sciences (for example, the Reactor Experiment for Neutrino Oscillation (RENO) or the dark matter or neutrinoless double-beta decay experiments at the Yangyang Underground Laboratory), but the heavy bias towards applied physics remains.

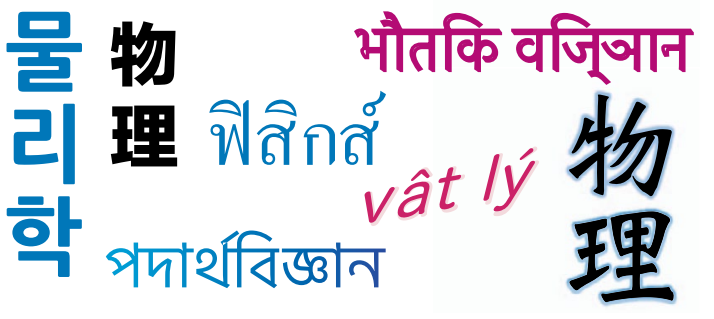

Credit: Charlotte Gurr/Springer Nature Limited
Another issue emphasized by the Viewpoint is that both researchers and editors are frustrated with the low numbers of scientists from Asia involved in the peer review process. It seems that the solution to this problem requires efforts from both sides: editors should try to identify and interact with more experts from Asia, whereas scientists could help by creating and keeping personal and group webpages up to date. Name disambiguation can also be problematic for anyone looking for a specific researcher with a common name. Nature Research journals are trying to solve this problem by mandating ORCIDs for all corresponding authors, and the American Physical Society journals offer authors the option to include their names in their own language.

What else can we do? As editors, we need to be aware of our own unconscious biases regarding who makes a good author or referee. It is easy to fall into the trap of unconsciously going back to the same people one has worked with before and therefore know to be reliable. We should challenge our own choices to ensure that they are fair. For example, editors need to ask themselves whether there are equally well-qualified experts to comment on a manuscript, who are not necessarily as visible as the most obvious choice of a referee.

We should also be on the watch for potential referee biases and unfair comments regarding English language proficiency. As one of the Viewpoint authors points out, such situations seem to happen more often when Asian authors are involved. Indeed, we have encountered unjustified, harsh comments made by referees regarding the English ability of authors from certain regions. Such gratuitous criticisms can negatively affect authors and undermine their confidence in submitting their work to some journals. Although English language proficiency is never a deciding factor in the editorial outcome, we will take a stance against such situations by reminding our referees that improving the language is, after all, our job.

But above all, what we need is to build a relationship with individuals and segments of the scientific community. Doing so is a long-term endeavour that takes time and patience, but at the same time it is mutually rewarding. We will start by interacting more in person with physicists from Asia - next time one of our editors is at a conference, let's have a chat. 